\title{
A contribution to the technique of intracutaneous testing with toxoplasmin
}

\author{
OTTO JÍROVEC AND JIND R̆ICH JÍRA \\ From the Protozoological Laboratory of the Czechoslovak Academy of Sciences, \\ Prague, Czechoslovakia
}

SYNOPSIS The preparation and application of toxoplasmin for intradermal testing are described. The persistences of sensibility to toxoplasmin and the specificity of the test are discussed.

A great deal of attention has been directed to acute toxoplasmosis, congenital and acquired, and much less to chronic and latent infections. These cannot readily be investigated by the direct methods of demonstrating the parasite in histological sections or by isolating it by animal inoculation. Recourse has to be had to indirect, antigen-antibody reactions. In addition to the Sabin-Feldman dye test, the complement-fixation test, and the haemagglutination test, we use the intradermal test with toxoplasmin. Over the past six years we have performed this test on 15,000 persons. This paper describes the preparation and use of the antigen. We intend to report in a subsequent paper the results obtained of tests performed on normal and other groups of the population.

\section{PREPARATION OF ANTIGEN FOR THE} INTRADERMAL TEST

The main modifications on the original method of Frenkel (1948) are that we do not use ultra-violet light, because it may destroy proteins, and we do not add heparin.

Fifty to sixty mice of about $20 \mathrm{~g}$. weight, from stocks known to be free from salmonellosis and ectromelia, were inoculated intraperitoneally with $0.1 \mathrm{ml}$. of a $1: 10$ to $1: 20$ dilution in saline of fresh mouse peritoneal exudate containing large numbers of toxoplasma (Bk strain). Four days later the mice were killed, and peritoneal exudate aspirated with aseptic precautions. Each mouse yielded from 0.2 to $1.0 \mathrm{ml}$. The exudates from groups of 10 mice were pooled in sterile tubes. Tests for bacterial sterility were set up. Then the material was centrifuged at 3,000 r.p.m. for 30 minutes and the supernatant fluid discarded. The deposit, consisting of toxoplasma and white blood corpuscles, was stored at $-20^{\circ} \mathrm{C}$. until the results of the sterility test were known. Then it was added in about $3 \mathrm{~g}$. amounts to $3 \mathrm{ml}$. of normal saline containing $0.25 \%$ of phenol. The mixture was

Received for publication 24 January 1961. homogenized in a Potter-Elvehjem all-glass tissue grinder for five minutes, after which it was thrice frozen to $-70^{\circ} \mathrm{Cr}$ and thawed. It was then diluted $1: 10$ with phenol saline and stored in a $4^{\circ} \mathrm{C}$. refrigerator for three weeks, during which time it was shaken daily. After this it was centrifuged, and the supernatant fluid removed. This was thrice frozen and thawed and then centrifuged to get rid of particulate matter, which would otherwise precipitate and have no antigenic value. The antigen thus prepared is stored in the refrigerator and diluted from time to time as required.

The antigen is tested for sterility and absence of toxicity or living toxoplasma by the State Institute for the Control of Drugs. The latter test is done by inoculating mice in three successive passages. Up to the present no toxic reactions have been observed in mice inoculated with the dilutions used for man.

TITRATION OF ANTIGEN The dilution at which the antigen is used is the highest which gives a characteristic, welldefined reaction in an allergic person. Much depends on how the antigen is made, particularly its homogenization. Early batches could not be diluted beyond $1: 200$ to $1: 500$. Current batches will stand dilution of $1: 1,000$ to $1: 2,000$ giving identical intensity of cutaneous reaction. Each fresh batch is tested in parallel with the previous batch on 10 to 15 negatively-reacting and on the same number of positively-reacting persons.

Tests on 104 persons have shown that the cell-free portion of toxoplasma containing mouse peritoneal exudate has only weak antigenicity in dilutions of $1: 5$ to $1: 100$.

TECHNIQUE OF THE INTRADERMAL TEST

We inoculate the control solution, phenol saline, into the cleansed skin above the insertion of the deltoid muscle and the antigen below the insertion. These sites have been chosen as being as sensitive as and less painful than the anterior aspect of the forearm. Instead of judging the amount of inoculum by the quantity expelled from the syringe, which is subject to loss through leakage, we do so by the size of wheal produced, which should measure 
$8 \mathrm{~mm}$. in diameter. Usually this is produced by 0.07 to $0.08 \mathrm{ml}$. of antigen.

In a very large number of tests we have had no serious reactions. Rarely there are general symptoms such as evening fever, swelling of lymph glands, especially in the axillae and groins, chills, and vomiting. Occasionally necrosis has developed at the site of infection but healed within 10 days. In one patient a psoriasis-like eruption developed. Care should be taken in the inoculation of patients with ocular inflammations, particularly chorioretinitis, for they may have an allergic exacerbation, which can, however, be suppressed by cortisone. We would emphasize that toxoplasmin is thermostable. Syringes used for controls should be kept separately and carefully rinsed.

\section{READING OF TEST}

The test is read after 24 hours and again after 48 hours. Rarely a person negative at 24 hours develops a reaction after $\mathbf{4 8}$ hours. In such cases the maximum reaction does not appear till 72 hours. All negatives should be examined again after 72 hours. The size of erythema and induration is measured in millimetres in two cross diameters and scored as in Table $\mathrm{I}$. We consider reactions read as + of no significance and $\mathrm{a}++$ reaction of doubtful significance.

\section{TABLE I}

EVALUATION OF THE TOXOPLASMIN TEST

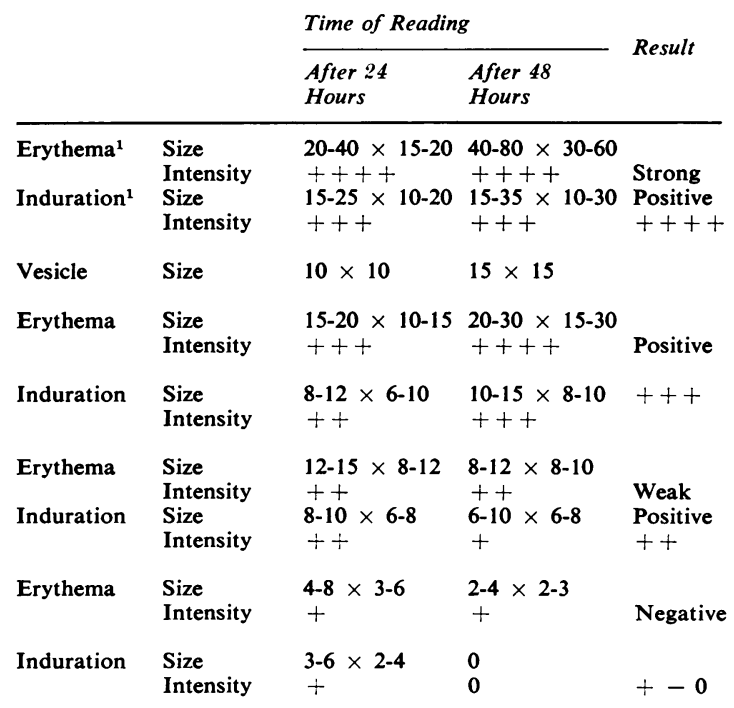

${ }^{1}$ Size of erythema and induration in $\mathrm{mm}$.

DEVELOPMENT AND PERSISTENCE OF SENSITIVITY TO TOXOPLASMIN

We re-examined three groups at intervals of one to four years with the toxoplasmin test.
1 Of 108 persons negative at the first test, $165(91.7 \%)$ remained negative, five $(2 \cdot 8 \%)$ became weakly positive, and $10(5.5 \%)$ clearly positive. Two of the latter worked with laboratory animals, which had not, however, been intentionally infected with toxoplasma. One of these, as soon as her illness was clinically obvious, already had a dye test titre of $1: 16,000$, a complement-fixation titre of $1: 160$, and a strongly positive intradermal test. In another three cases the complement-fixation test became positive before the toxoplasmin skin test. In yet another case, not included in this series, a mother immediately after the delivery of a foetus proved to have toxoplasmosis, gave a negative complement-fixation test and skin test. Three months later a complement-fixation test was positive but not till eight months after delivery did a skin test become positive.

2 Of 130 persons weakly positive $(++)$ at the first test, $53(40.8 \%)$ gave the same reaction, $32(24.6 \%)$ gave a negative reaction, and $45(34.6 \%)$ gave a clearly positive reaction.

We believe that those who were first ++ and then negative had shown a non-specific reaction.

3 Of 363 persons strongly positive $(+++$ or $++++)$ at the first test, $208(57 \cdot 3 \%)$ remained the same, $44(12.1 \%)$ fell from ++++ to,$+++ 52(14.3 \%)$ fell from +++ to ++ , and $59(16.3 \%)$ rose from +++ to ++++ . We have not observed an original +++ or ++++ become negative.

Persistence of skin sensitivity has been proved in one instance for 37 years (the mother of the first case ever described, by Janků in 1923); in two for nine years (in one of these instances the infected daughter survives and is also positive), and in another for five years.

\section{SPECIFICITY OF THE TOXOPLASMIN INTRADERMAL TEST}

It might be objected that the reactions were due to nonspecific material. To meet this we prepared and tested toxoplasmins from $(a)$ infected mouse peritoneal exudate, (b) infected chick-embryo chorio-allantoic membrane, (c) infected tissue culture and at the same time, as controls, we tested similar but non-infected preparations.

Chick-embryo toxoplasmin was prepared by inoculating the chorio-allantoic membranes of 10-11-day-old embryos with 0.1 to $0.2 \mathrm{ml}$. of a $10 \%$ suspension of infected mouse peritoneal exudate or of infected chorio-allantoic membrane. Four days later the whole egg membranes, or those parts that showed plaques due to toxoplasma growth, were removed, tested for bacteriological sterility, and kept for several days at $-20^{\circ} \mathrm{C}$. They were then washed in saline, finely chopped, homogenized in a M.S.E. homogenizer, three times frozen $\left(-70^{\circ} \mathrm{C}\right.$.) and thawed $\left(37^{\circ} \mathrm{C}\right.$.), and again homogenized. Phenol saline was then added; the subsequent procedure was as for mouse peritoneal exudate toxoplasmin. As the chorioallantoic membrane antigen contained relatively less toxoplasma material than the mouse peritoneal exudate antigen it was used in lower dilution (1:150 to $1: 200)$. Table II shows the results of comparative tests with the 


\section{TABLE II}

COMPARISON OF INTENSITY OF REACTIONS WITH MOUSE PERITONEAL EXUDATE TOXOPLASMIN AND CHICK EMBRYO CHORIO-ALLANTOIC MEMBRANE TOXOPLASMIN ON 390 PERSONS

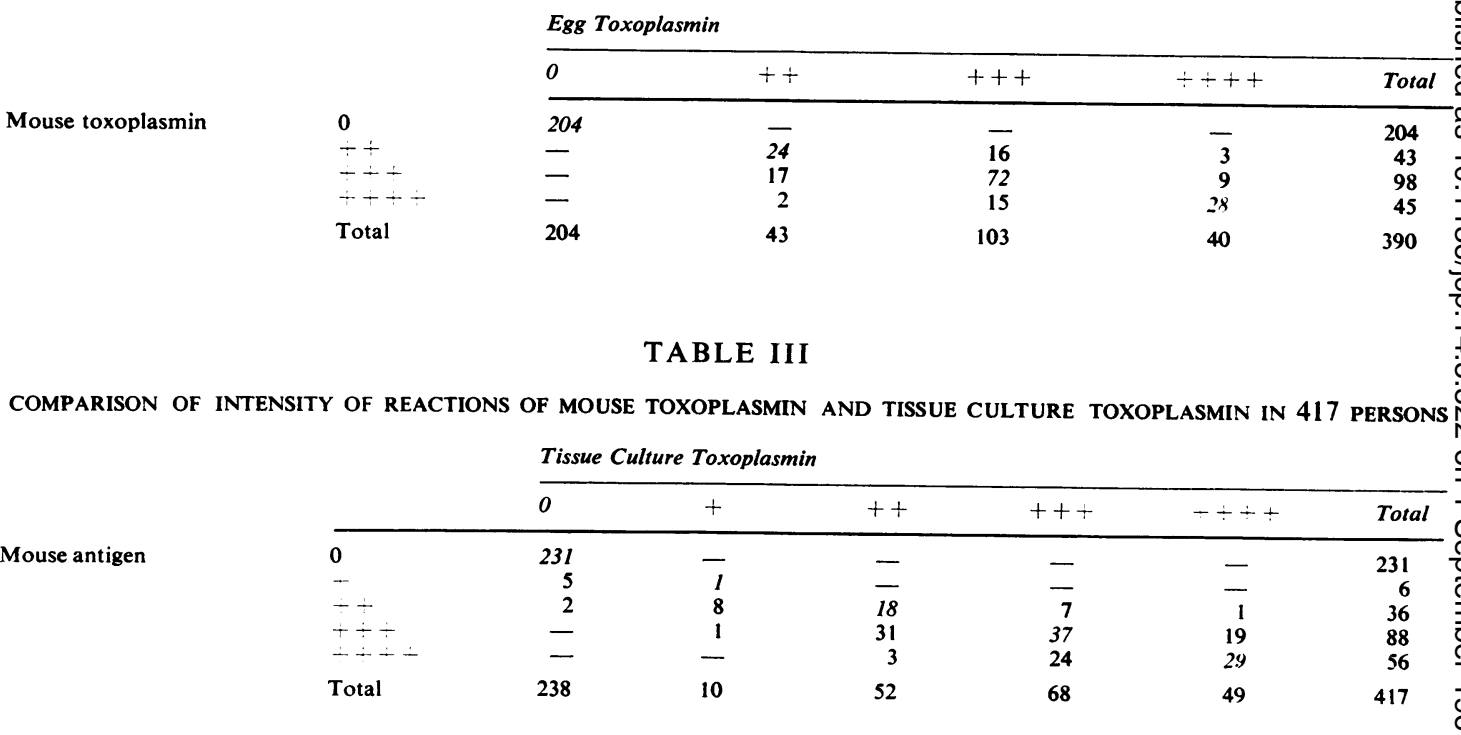

mouse peritoneal exudate antigen and the egg antigen (the latter at a dilution of $1: 200$ ). The results show agreement in 328 of the 390 tests.

Tissue culture toxoplasmin was prepared by infecting 6-day-old trypsinized monkey kidney cultures (in big Roux jars) with mouse peritoneal exudate containing toxoplasma. After seven days of culture the toxoplasmas were removed from the medium by centrifugation and toxoplasmin prepared as from mouse exudate. The potency of the tissue culture antigen was lower than that of the mouse antigen. It was used in dilutions of $1: 50$ to $1: 300$.

Table III shows the results of comparative tests with the mouse antigen and the tissue culture antigen. Complete agreement was found in 316 persons $(75.8 \%)$. A really significant difference was found in one case only $(+++$ with mouse antigen, + with tissue culture antigen). The other differences do not exceed the variations in intensity of reaction to be expected in such a test, for it is impossible to inoculate intradermally absolutely identical amounts of antigen.

The control preparations used and the results obtained with these were as follows:-

1 Sterile ascitic fluid artificially produced in healthy mice was used to test 32 persons. None reacted. 2 Extract of mouse spleen diluted 1:500 was used to test 560 persons. Only 11 reacted and they gave no more than + insignificant reactions. 3 Chorio-allantoic membrane of 14-15-day-old chick embryos diluted $1: 200$ was used to test 326 persons. None reacted. 4 Non-infected tissue culture was used to test 326 persons. None reacted. 5 Phenol saline was used to test 12,280 persons and two gave a + reaction.

\section{APPLICATIONS OF THE TOXOPLASMIN TEST}

Since the toxoplasmin test is easy to perform and sensitization with positive reaction persists for long periods, it is well suited to estimating the amount of toxoplasma infection there is, or has been, in a community.

It is also of value in assessing the part played by toxoplasmosis in the production of various diseases, congenital and acquired. Thus it can be used in the investigation of the aetiology of congenital developmental defects-mongolism, oligophrenia, and so on. When this is done it is important to test not only the children but also their mothers. The evidence obtained is statistical, hence it is necessary (1) to test a homogeneous control group of approximately the same age, from the same locality, similarly employed; (2) to obtain a careful and detailed clinical diagnosis on the individual members of the test group, e.g., to differentiate granulomatous from other forms of uveitis (toxoplasmosis has thus been found to play a part in the former but not in the latter); to exclude individuals whose illness is shown to be due to another cause; to have sufficient numbers for statistical analysis.

\section{REFERENCES}

Frenkel, J. K. (1948). Proc. Soc. exp. Biol. (New York), 68, 634. Jankú, J. (1923). Cas. Lék-ces., 39-43. 26 (German translation in Cse. Parasit. 1959, 6, 1). 